\title{
SEQUENCE ANALYSIS OF Streptococcus agalactiae, A PATHOGEN CAUSING STREPTOCOCCOSIS IN TILAPIA (Oreochromis niloticus)
}

\author{
Angela Mariana Lusiastuti"\#, Taukhid"), Eny Kusrini"**), and Wartono Hadie ${ }^{* * * *}$ \\ ") Research Institute of Freshwater Aquaculture, Bogor \\ *M) Research Station of Ornamental Freshwater Aquaculture \\ Jl. Perikanan No. 13, Pancoran Mas, Depok 16436 \\ ****) Research Center Indonesia Aquaculture \\ Jl. Ragunan 20 Pasar Minggu, Jakarta Selatan 12540
}

\begin{abstract}
Pathogen identification based on biochemical properties can barely differentiate Streptococcus iniae and S. agalactiae. Beside that, this technique is also limited by the length of time required to complete the assays. Therefore, rapid diagnosis is necessary to initiate prompt therapeutic and prophylactic measures in order to limit any potential economic losses caused by such pathogens. The aim of the present study was to identify Streptococcosis species using amplification of S. agalactiae DNA sequence with species-specific primer Sdi 61 AGGAAACCTGCCATTTCCG and Sdi 252 CAATCTATTTCTAGATCGTGG and perform phylogenetic analysis based on DNA nucleotide sequence data. The sequencing of PCR products was performed at BPPT Puspiptek Serpong by using the respective PCR primers, Big Dye Terminator Chemistry and AmpliTaq-FS DNA polymerase. The sequencing reactions were run on the ABI Prism version 3103 - Avant Genetic Analyzer (USA) and the result was read by Sequence Navigator program (Applied Biosystem). Alignment multiple analysis was done based on the data from Genebank with BLASTN (http://blast.ncbi.nlm.nih.gov/ blast.cgi) on the nucleotide level. Neighbor-joining phylogenetic trees were generated with Genetyx programme version 7 with UPGMA and MEGA software version 4.0. The result revealed that the isolates from brain, eye, and kidney of diseased Tilapia were infected by S. agalactiae and it has $99 \%$ similarity with Genebank. It has close relationship with $S$. agalactiae at genebank with UPGMA method. These isolates showed high variation in the first sequence which is similar to $S$. iniae. The information of $S$. agalactiae genomes suggests that gene acquisition, duplication, and reassortment have played an important role in genetic diversity and evolution of $S$. agalactiae. Screening of breeder fish stocks with the developed PCR methodology, followed by elimination of infected stocks, would provide an efficient strategy to control fish infected by streptococcosis.
\end{abstract}

KEYWORDS: Streptococcus agalactiae, tilapia, sequence analysis

\section{INTRODUCTION}

Tilapia infected by Streptococcosis ( $S$. iniae and S. agalactiae) exhibits very similar symptoms and clinical signs regardless of the etiologic agents (Kusuda \& Salati, 1999), there- fore a definitive diagnosis of the etiological agent was based on the microbiological characteristics of bacteria isolated from diseased fish (Mata et al., 2004). Recently, molecular methods based on DNA probes or PCR have overcome the problems associated with cul-

\# Corresponding author. Research Institute for Freshwater Aquaculture. Jl Sempur No. 1, Bogor, Indonesia. Tel.: + 622518327890

E-mail address: Iusiastuti@yahoo.com 
ture-based techniques, enabling the detection of microorganisms directly from clinical samples without isolation (Gonzalez et al., 2004). Moreover, Gonzales et al. (2004) stated that molecular techniques have been the most effective methods in diagnosing pathogens because they are more specific and sensitive than other conventional or commercial assays. The detection of pathogens using PCR and continuing sequence analysis is faster and cost-effective. The aim of the present study was to identify streptococcosis species by amplification of $S$. agalactiae DNA sequence with species-specific primer Sdi 61 AGGAAACCTGCCATTTGCG and Sdi 252 CAATCTATTTCTAGATCGTGG and perform phylogenetic analysis based on DNA nucleotide sequence data.

\section{MATERIALS AND METHODS}

\section{S. agalactiae isolates}

Isolates containing suspected $S$. agalactiae was obtained from Cirata Reservoir, West Java, and Wadas Lintang reservoir, Central Java Province. As part of this program, $S$. agalactiae strains were isolated from diseased Tilapia (Oreochromis niloticus). The isolates were stored at $-20^{\circ} \mathrm{C}$ in brain heart infusion (BHI) broth (Becton Dickinson, Sparks, Md.) with $15 \%$ glycerol.

\section{Bacterial DNA isolation}

The samples of bacterial DNA used in PCR was extracted using boiling method. Bacterial aDNA was obtained from cells by touching the colonies and grown on blood agar medium with sterile ose. Bacterial colonies (5-10 colonies) were suspended in $400 \mu$ l of RNAse free water. The sample was boiled for $10 \mathrm{~min}$ at $98^{\circ} \mathrm{C}$ and centrifuged at $8.000 \times \mathrm{g}$ for $10 \mathrm{~min}$. The supernatant was then used for PCR amplification.

\section{PCR amplification}

A set of oligonucleotide primers was designed to amplify 192 base pairs from gene target $16 \mathrm{~S}$ intergenic spacer of S. agalactiae (spesies-spesific primers) (Mata et al., 2004). These primers were designated as Sdi 61: 5'AGGAAACCTGCCATTTGCG-3' and Sdi 252: 5'CAATCTATTTCTAGATCGTGG-3' (Alpha DNA Montreal, Quebec). The process of PCR ampli- fication was performed in $25 \mu \mathrm{L}$ tube containing: $12.5 \mu \mathrm{L}$ master mix GoTaq ${ }^{\circledR}$ Green (Promega, Madison WI USA), $8.5 \mu \mathrm{L}$ nuclease free water, 1 $\mu \mathrm{L}$ primers (reverse and forward) each and $2 \mu \mathrm{L}$ DNA templates. The amplification cycle at thermal cycler T personal (Biometra) consisted of 2 min denaturation at $94^{\circ} \mathrm{C}$ followed by 25 cycles of denaturation at $92^{\circ} \mathrm{C}$ for $1 \mathrm{~min}$, annealing at $55^{\circ} \mathrm{C}$ for $1 \mathrm{~min}$, and elongation at $72^{\circ} \mathrm{C}$ for $90 \mathrm{~s}$. Finally, the last elongation was done at $72^{\circ} \mathrm{C}$ for $5 \mathrm{~min}$. RNAse free water was used as negative control (no DNA template).

\section{PCR and DNA sequencing}

Cell lysates for PCR were prepared using lysozyme and proteinase $\mathrm{K}$ as described previously. Primers Sdi 61: 5'AGGAAACCTGCCATTTGCG-3' and Sdi 252: 5'CAATCTATTTCTAGATCGTGG-3' (Alpha DNA Montreal, Quebec) were used from known sequences (GenBank accession number AF064441.1) to amplify the 192-bp fragment. PCR conditions consisted of an initial 5-min denaturation step at $95^{\circ} \mathrm{C}, 30$ cycles of $95^{\circ} \mathrm{C}$ for $30 \mathrm{sec}, 55^{\circ} \mathrm{C}$ for $30 \mathrm{sec}$, and $72^{\circ} \mathrm{C}$ for 30 $\mathrm{sec}$, and a final 10 -min extension cycle at $72^{\circ} \mathrm{C}$. PCR products were purified using the QIAquick PCR purification kit (QIAGEN, Inc., Chatsworth, Calif.). Following gel electrophoresis, DNA was quantified by using the Lablmage gel analysis software (version 2.62; Kapelan, Saale, Germany) to compare the band intensities of PCR products to marker band intensities of known concentrations (pGEM marker; Promega, Madison, Wis.). The sequencing of PCR products was performed at BPPT Puspiptek Serpong by using the respective PCR primers, Big Dye Terminator chemistry, and AmpliTaq-FS DNA polymerase; the sequencing reactions were run on the $A B I 3700$ automated sequencer (Perkin-Elmer Biosystems, Foster City, Calif.). DNA sequence data obtained with both forward and reverse primers were proofread and assembled with the Seqman software program (DNAStar, Madison, Wis.) to yield the final $16 \mathrm{~S}$ rRNA sequences used for analyses. $16 \mathrm{~S}$ rRNA sequences were aligned using BIO software and multiple aligment analysis was used to assign it with sequences data from Genebank with BLASTN (http://blst.ncbi.nlm.nih.gov/ blast.cgi) at nucleotide level. Neighbor-joining phylogenetic trees were generated with MEGA software version 4.0 and Genetyx software version 7 with UPGMA method. 


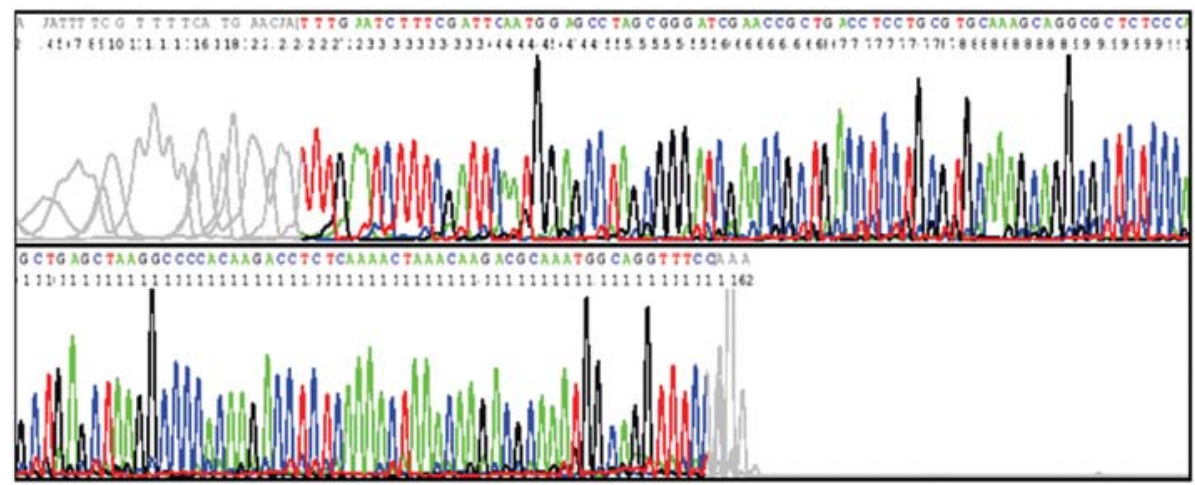

CGAAACTGAGAGGTCTTGTGGGGCCTTAGCTCAGCTGGGAGAGCGCCTGCTTTGCACGCA GGAGGTCAGCGGTTCGATCCCGCTAGGCTCCATTGAATCGAAAGATTCAAATTGTTCATTG AAAATTGAATATCTATATCAAATTCCACGATCTAGAAATAGTAGACAAGTGAGCTAAGGCCC CACAAGACCTCTCAAAACTAAACAAGACGCAAATGGTAAAGTTTAC

TAAATTTTCGTTTTCATGAACAATTTGAATCTTTCGATTCAATGGAGCCTAGCGGGATCGAA CCGCTGACCTCCTGCGTGCAAAGCAGGCGCTCTCCCAGCTGAGCTAAGGCCCCACAAGA CCTCTCAAAACTAAACAAGACGCAAATGGCAGGTTTCCAAA

Figure 1. Chromatogram (top) and sequencing result of 16S rRNA of S. agalactiae (down)

\section{RESULT AND DISCUSSION}

\section{Sequence Similarity}

Phylogenetic analysis of the $16 \mathrm{~S}$ rRNA gene sequence clearly revealed that $S$. agalactiae is a member of the pyogenic group of the genus Streptococcus. The closest relative is $S$. difficilis, with which it shares almost identical sequence (99\%). S. agalactiae and $S$. difficilis constitute a cluster that was separate from the other Streptococcus species.

The multiple alignment sequence data of 16S rRNA was similar to those of some species of S. agalactiae especially S. difficilis available at Genebank (Figure 2). The number of nucleotide between $S$. agalactiae and $S$ agalactiae from Genebank is 192 bp. Kawamura et al. (2004) found that S. agalactiae has high genetic similarity with $S$. difficilis. Based on their research, it was proposed that $S$. difficilis is a later synonym of $S$. agalactiae.

However, different sequence was found in S. agalactiae and S. iniae, showed in Figure 3. Multiple alignments between S. agalactiae and S. iniae described a high variation for the first sequence.
Comparative phylogenetic tree was constructed from $16 \mathrm{~S}$ rRNA gene sequences. Distances were calculated by using the neighbor-joining method. S. agalactiae from this research were closely related, with very little sequence divergence with $S$. agalactiae from the Genebank. Elliot et al. (1990) showed that strains isolated from humans, mice and fish shared identical whole cell protein profiles. These reports strongly suggest that the majority of strains of group B streptococci isolated from different hosts belong to a single cluster.

The broad spectrum of $S$. agalactiae disease manifestations reflects a complex interplay between the host's innate and adaptive immune systems and bacterial virulence factors. On the other hand, S. agalactiae is part of normal flora, and thus an opportunistic pathogen. Therefore, the role of host and environment is very important for the occurrence of streptococcosis disease. S. agalactiae virulence traits mainly include: (a) factors that inhibits immunological clearance; (b) adhesion or invasion that enables penetration of epithelial and endothelial cellular barriers to reach the bloodstream and deeper tissues; (c) toxins that directly injure or disrupt host tissue components. 

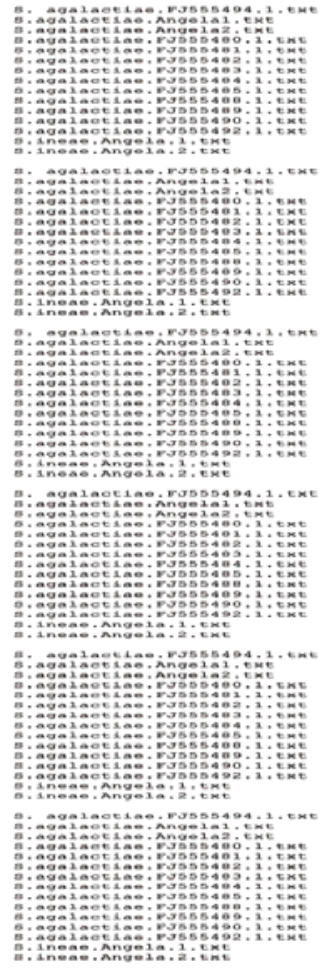

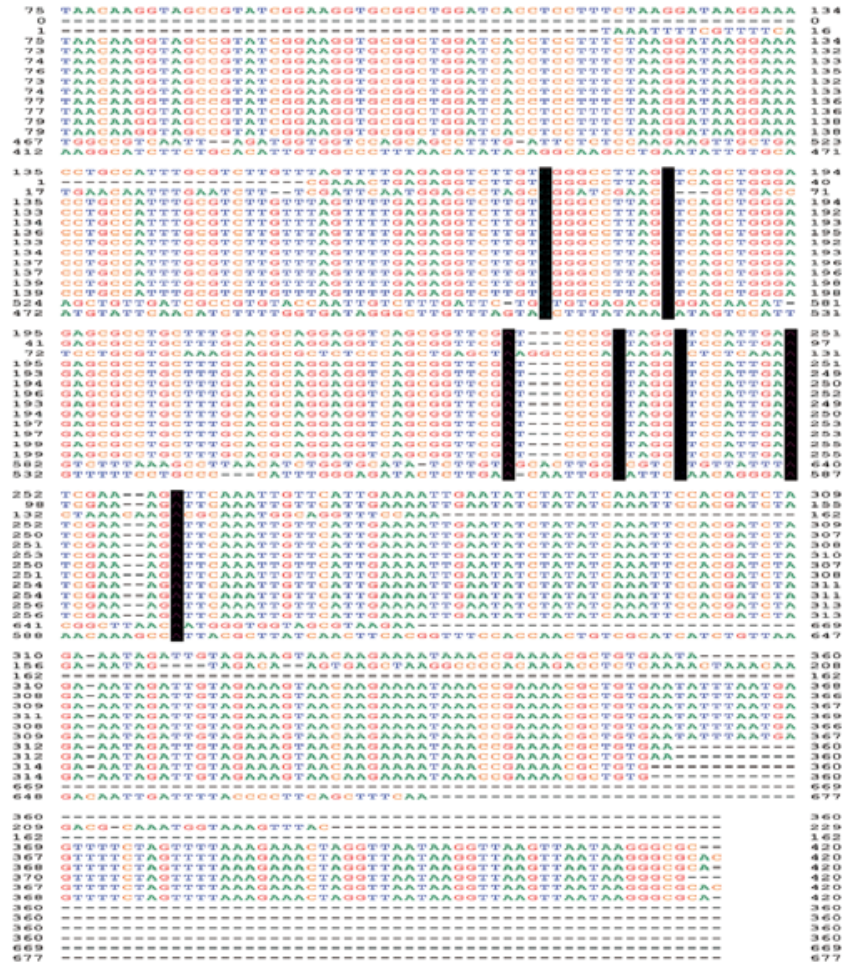

Figure 2. Multiple alignments of 192 bp fragment from 16S rRNA S. agalactiae and. S. iniae and S. agalactiae from Genebank
S. agalactiae.FJ555494.1.txt S.agalactiae. Angela1.txt

S.agalactiae.FJ555480.1.txt S.agalactiae.FJ555481.1.txt S.agalactiae.FJ555482.1.txt S.agalactiae.FJ555483.1.txt S.agalactiae.FJ555484.1.txt S.agalactiae. FJ555485.1.txt S.agalactiae.FJ555488.1.txt S.agalactiae.FJ555489.1.txt S.agalactiae.FJ555490.1.txt S.agalactiae.FJ555492.1.txt

S.ineae.Angela.1.txt

S.ineae.Angela.2.txt

S. agalactiae.FJ555494.1.txt S. agalactiae.Angela1.txt S.agalactiae.Angela2.txt S.agalactiae.FJ555480.1.txt S.agalactiae.FJ555482.1.txt S.agalactiae.FJ555483.1.txt

S.agalactiae.FJ555484.1.txt S.agalactiae.FJ555485.1.txt S.agalactiae.FJ555488.1.txt S.agalactiae.FJ555489.1.txt S.agalactiae.FJ555490.1.txt S.agalactiae.FJ555492.1.txt

S.ineae.Angela.1.txt

S.ineae.Angela.2.txt S.agalactiae. Angela2.txt S.agalactiae.FJ555481.1.txt

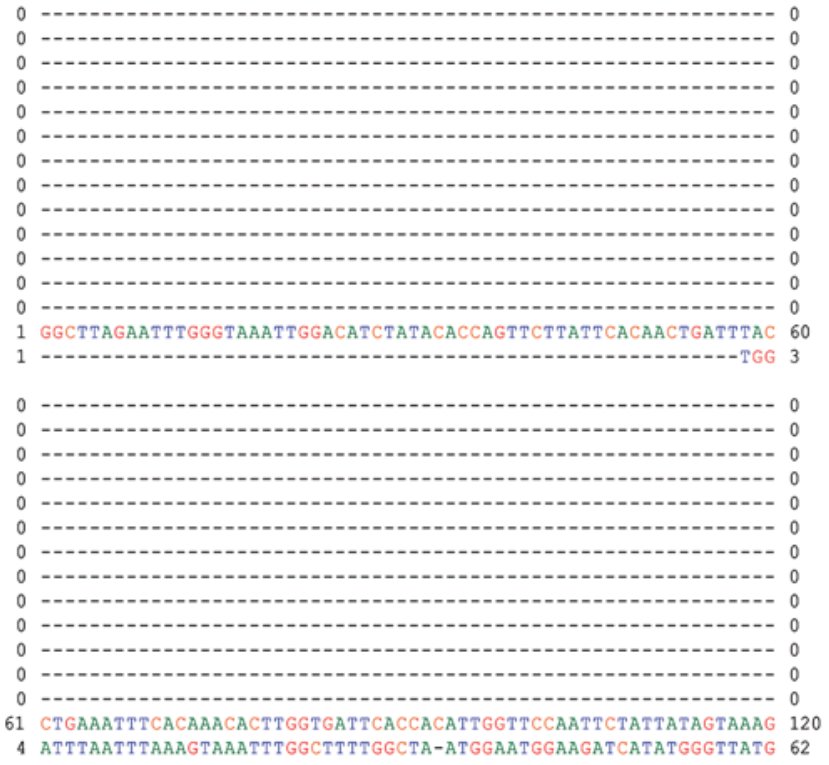

Figure 3. Multiple alignments of $S$. agalactiae and $S$. iniae. The dot means the nucleotide is identical with the genetic sequence above 


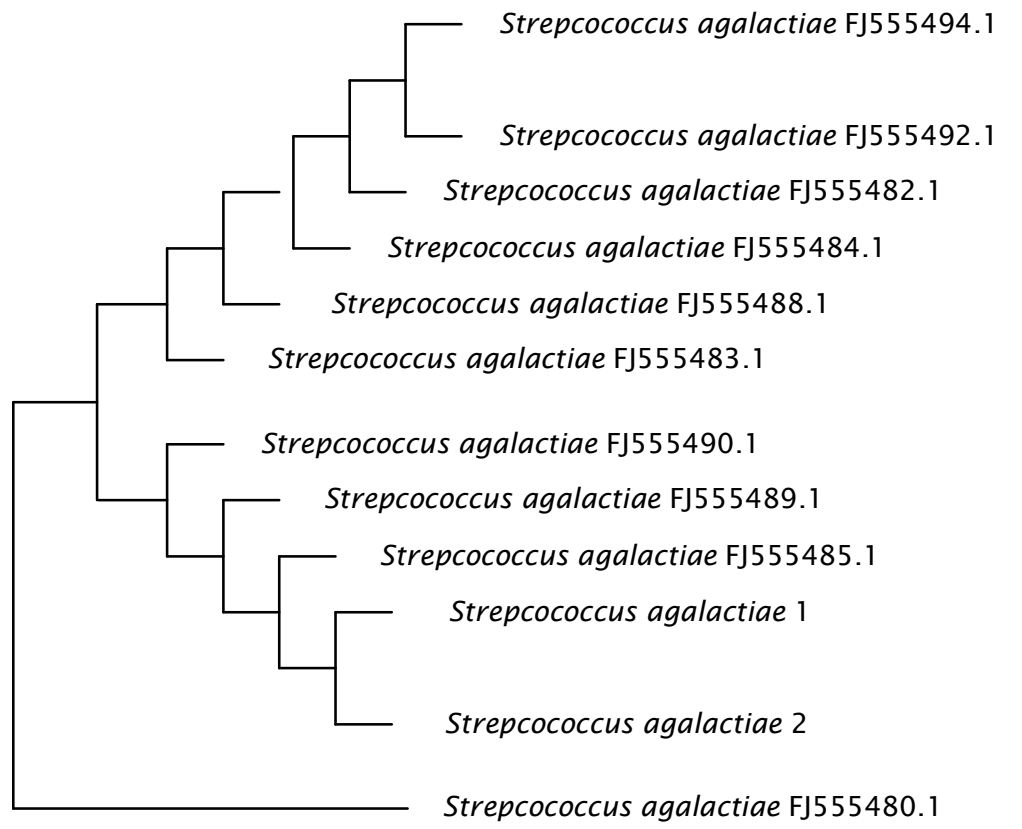

Figure 4. Dendrogram UPGMA based on nucleotide 16S rRNA S. agalactiae and 192 bp S. agalactiae from Genebank

Capsular serotyping has been the classic method used in descriptive epidemiology of S. agalactiae. Based on capsular expression, $S$. agalactiae are sub-classified into nine serotypes, Ia, Ib, and II - VIII. In 2002, two complete genome sequences of $S$. agalactiae were released for a serotype III strain and a serotype V strain (Tettelin et al., 2002). The genome sequences of additional six S. agalactiae invasive strains were reported in 2005 , which include serotype Ia, Ib, II, III, and V (Tettelin et al., 2002). The S. agalactiae genome is around $2 \mathrm{Mb}$ with low $\mathrm{G} / \mathrm{C}$ content of about $36 \%$, and is predicted to comprise more than 2,100 protein-encoding genes. Of these encoding genes, there have been 14-15 pathogenicity island-like regions identified, which are dispersed around the genome. These "islands" contain encoding genes and putative virulence genes, mostly predicted surface proteins. The "islands" may have an important role in acquisition of virulence factors and in genetic diversification of the species (Tettelin et al., 2002).

Importantly, S. agalactiae appears to have a rather large flexible gene pool, and an average of 33 new strain-specific genes is pre- dicted to be identified when a new $S$. agalactiae strain is sequenced (Tettelin et al., 2002). The information of S. agalactiae genomes suggests that gene acquisition, duplication, and reassortment have played an important role in genetic diversity and evolution of S. agalactiae.

Considering the data presented here and data from other researchers, we conclude that the isolates from Cirata and Wadas Lintang, Java Province are S. agalactiae and distinguishable from S. iniae particularly from genetical aspects.

\section{REFERENCES}

Elliot, J.A., Facklam, R.R., \& Richter, C.B. 1990. Whole-Cell Protein Patterns of Nonhemolytic Group B, Type 1b, Streptococci Isolated from Humans, Mice, Cattle, Frogs, and Fish. J Clin Microbiol, 28: 628-630

Kawamura, Y., Itoh, Y., Mishima, N., Ohkusa, K., Kasai, H., \& Ezaki, T. 2004. High Genetic Similarity of Streptococcus agalactiae and Streptococcus difficilis: S. difficilis Eldar et al. 1995 is a Later Synonym of S. agalactiae Lehmann and Neumann 1896 (Approved List 1980). DOI 10.1099/ijs.o.63403-0. Published on line 12 November 2004. 
Kusuda, R. \& Salati, F. 1999. Nterococcus seriolicida sp. a fish pathogen. Int. J. Sys. Bacteriol, Al : 406 - 409.

Mata, A.I., Blanco, M.M., Domingeus, L., Fernandes - Garayzabal, J.F., Gibello, A. 2004. Development of PCR assay for streptococcus iniae based on the lactate oxidase gene with potential diagnostic value. vet. microbiol., 101: 101-116.

Gonzales, S.F.M., Krug, J., Nielson, M.E., Santos, Y., Call, D.R. 2004. Simultaneous detection of marine fish pathogens by using multiplex PCR and DNA Microarray. J. Clin. Microbiol., 42 : 1414 - 1419.

Tettelin, H., V. Masignani, V., Cieslewicz, M.J., Eisen, J.A., Peterson, S., Wessels, M.R.,
Paulsen, I.T., Nelson, K.E., Margarit, I., Read, T.D., Madoff, L.C., Wolf, A.M., Beanan, M.J., Brinkac, L.M., Daugherty, S.C., DeBoy, R.T., Durkin, A.S., Kolonay, J.F., Madupu, R., Lewis, M.R., Radune, D., Fedorova, N.B., Scanlan, D., Khouri, H., Mulligan, S., Carty, H.A., Cline, R.T., Van Aken, S.E., Gill, J., Scarselli, M., Mora, M., Lacobini, E.T., Brettoni, C., Galli, G., Mariani, M., Vegni; F., Maione; D., Rinaudo; D., Rappuoli, R., Telford, J.L, Kasper, D.L., Grandi, G., \& Fraser, C.M. 2002. Complete Genome Sequence and Comparative Genomic Analysis of An Emerging Human Pathogen, Serotype V Streptococcus agalactiae. Proc. Natl. Acad. Sci. USA, 99: 12391-12396. 